\title{
Eye Movement Monitoring and Maturation of Human Face Exploration
}

\author{
Nadia Aguillon-Hernandez ${ }^{a, b}$ Laëtitia Roché ${ }^{b}$ Frédérique Bonnet-Brilhault ${ }^{a-c}$ \\ Sylvie Roux ${ }^{a, b}$ Catherine Barthelemy ${ }^{a-c}$ Joëlle Martineau ${ }^{a, b}$ \\ aUnité Mixte de Recherche, Inserm U 930, b Université François-Rabelais de Tours, and 'Service de Pédopsychiatrie, \\ Hôpital Bretonneau, CHRU de Tours, Tours, France
}

\section{Key Words}

Eye tracking $\cdot$ Development $\cdot$ Face $\cdot$ Emotion

\begin{abstract}
Objective: The aim of this study was to characterize ocular exploration of neutral and emotional faces in the typical development of a child. Subjects and Method: In this eye-tracking study, visual exploration of faces (with neutral or emotional expressions: happiness or sadness) was characterized in a population of 52 children ( 24 girls and 28 boys from 4 to 15 years of age) and 44 adults ( 22 women and 22 men from 18 to 35 years of age). The time spent on the eyes, nose and mouth of the faces was measured. Results: All participants spent more time on the eyes (13\%) rather than the nose and mouth (6\%). The youngest participants spent less time exploring the eyes than the older participants, suggesting the progressive establishment of interest in these informative regions of the face during maturation. This process seemed to occur later in females (7-9 years) than males (4-6 years). Conclusion: These results confirm the importance of the eye area and the capacity of this region to capture attention. In addition, this study shows that the exploration of this region increases with age and is lower among girls aged 4-6 years compared with boys of the same age.
\end{abstract}

(c) 2016 S. Karger AG, Basel

\section{Introduction}

In humans, there is an early attention link to the face which allows social and emotional reciprocity, the basis of the development of social and emotional relationships [1, 2]. Healthy adults are 'experts' in the recognition of faces [3] while all faces present the same configuration, and the level of expertise partly determines the establishment of social competence. Face processing may be influenced by the age of the observer, and electrophysiological studies have shown that the response to faces varies with age [4]. So far, no study has been conducted to evaluate the exploration of the eyes in the perception of faces during development. For quantifying this exploration, eye-tracking devices can be used to precisely determine gaze direction [5] and to record ocular behavior, and thus better understand the initial stage of perception with the exploration of visual stimuli [5]. This mode of investigation is being used more and more frequently to study disorders characterized by abnormalities in ocular behavior such as autism [6-8]. All these studies have been performed in adults and children of both genders [9]. However, there are gender differences in development, especially in sociocommunicative development.

It is known that girls develop language [10] and theoryof-mind [11] skills earlier than boys. Moreover, girls develop social and structured forms of play at younger ages

Dr. Nadia Aguillon-Hernandez

UMR Inserm U 930, Imagerie et Cerveau, Equipe 1 - 'Autisme'

Université François-Rabelais de Tours, Bâtiment B1A, Bretonneau, CHRU

FR-37044 Tours Cedex 9 (France)

E-Mail nadia.aguillon@univ-tours.fr
This is an Open Access article licensed under the terms of the Creative Commons Attribution-NonCommercial 3.0 Unported license (CC BY-NC) (www.karger.com/OA-license), applicable to the online version of the article only. Distribution permitted for non-commercial purposes only. 
Table 1. Population size and average age (in years) of the male and female participants of the eye-tracking study

\begin{tabular}{|c|c|c|c|c|c|c|}
\hline & \multicolumn{2}{|c|}{ Male participants } & \multicolumn{2}{|c|}{ Female participants } & \multicolumn{2}{|c|}{ All participants } \\
\hline & $\mathrm{n}$ & mean age $\pm S D$ & $\mathrm{n}$ & mean age $\pm S D$ & $\mathrm{n}$ & mean age $\pm S D$ \\
\hline \multicolumn{7}{|l|}{ Children } \\
\hline 4-6 years & 8 & $5.3 \pm 0.9$ & 6 & $5 \pm 0.9$ & 14 & $5 \pm 1.2$ \\
\hline $7-9$ years & 8 & $7.5 \pm 0.8$ & 8 & $8.3 \pm 0.7$ & 16 & $7.9 \pm 0.8$ \\
\hline $10-12$ years & 8 & $10.9 \pm 0.8$ & 4 & $10.8 \pm 1$ & 12 & $10.9 \pm 0.8$ \\
\hline $13-15$ years & 4 & $13.5 \pm 0.6$ & 6 & $13.4 \pm 0.8$ & 10 & $13.7 \pm 1$ \\
\hline All children & 28 & $9.3 \pm 0.8$ & 24 & $9.4 \pm 0.9$ & 52 & $9.4 \pm 1$ \\
\hline \multicolumn{7}{|l|}{ Adults } \\
\hline $18-35$ years & 22 & $22.7 \pm 3.4$ & 22 & $21.5 \pm 1.7$ & 44 & $21.9 \pm 2.9$ \\
\hline
\end{tabular}

than boys [12]. Yet, face processing seems to be different between gender: in gender categorization tasks, it was reported that women were more efficient in recognizing faces and facial expressions than men $[13,14]$. Thayer and Johnsen [14] investigated recognition of facial emotional expressions based upon self-report ratings of felt emotion in healthy adults and found that females are more efficient compared to males during a gender task categorization. In their study, Cellerino et al. [13] used two different modes of spatial filtration, pixelation and Gaussian noise, and they tested healthy adults on face gender categorization. They found that male faces are categorized more efficiently than female faces and that participants are more efficient in categorizing same-sex faces [13]. These studies complement electrophysiological data showing that during basic visual perception significant differences between men and women in the amplitude of waves were recorded in the occipital region [15].

Due to the differences in neuroanatomical, cognitive, morphological, and biochemical levels between men and women, it is equally essential to evaluate the possible effects, depending on the different sexes, on ocular behavior. No study of eye tracking has been performed on the effects of gender on exploratory behavior during the perception of faces. The objective of this eye-tracking study was to characterize ocular exploration of neutral and emotional faces (sad and happy faces) in the typical development. In this eye-tracking study, we investigated the effects of maturation and gender during the exploration of expressed or unexpressed facial emotion in a population of typical adults and children. We postulated that visual exploratory behavior changes with age and that female and male subjects would explore a neutral male face differently throughout development.

Maturation of Face Exploration

\section{Material and Methods}

\section{Participants}

The study group consisted of two groups composed of 52 normally developing children and adults of both sexes; age ranged from 4 to 15 years for children and adolescents, and from 18 to 35 years for adults (table 1). The child study group was divided into four age categories: preschool children (4-6 years old), school children (7-9 years old), preadolescents (10-12 years old), and adolescents (13-15 years old). The group of normal adults consisted of 44 males and females. All participants were right-handed according to the Edinburgh criteria with laterality scores $\geq 0.8$ [16]. Written informed consent was obtained from each adult while that of the children was obtained from the parents. The study was approved by the Institution's Ethics Committee and conformes with the Code of Ethics of the World Medical Association [17].

Stimuli

A validated battery of neutral faces and faces expressing emotion (happy and sad faces) $[6,8]$ was used. The colors, background, position, and size of the faces of each image were harmonized.

The battery of neutral and emotional faces consisted of $30 \mathrm{col}-$ or photographs (on a beige background) of faces of men of European descent (18-35 years of age) with neutral, positive (happy), or negative (sad) expression, without distinctive signs (moustache, beard, scars, piercings); the study subjects had never seen them before. The same model appeared in the various emotional categories of the database, i.e. the same face was seen with a neutral expression, an expression of happiness, and an expression of sadness.

\section{Materials}

Visual stimuli were delivered by the head-free mounted faceLAB ${ }^{\circledR}$ eye-tracking system, which consists of a computer equipped with two digital infrared light cameras and an infrared light source (wavelength $875 \mathrm{~nm}$ ) derived from the international exposure standards set by the International Electrotechnical Commission [18]. The subject was not directly fitted with any equipment, and the corneal reflection of infrared light was used to monitor ocular behavior. GazeTracker ${ }^{\circledR}$ software was used to measure and analyze the duration of exploration on the face being observed (total face, mouth, nose, eyes). 
Fig. 1. Size and disposition of the different regions of interest and of anything outside the regions of interest. Areas called 'regions of interest' include the eye, nose, and mouth regions (left figure) and areas called 'outside regions of interest' (right figure) include the rest of the face (except the eye, nose, and mouth regions), the background image, and the area outside of the screen (not shown in the figure).

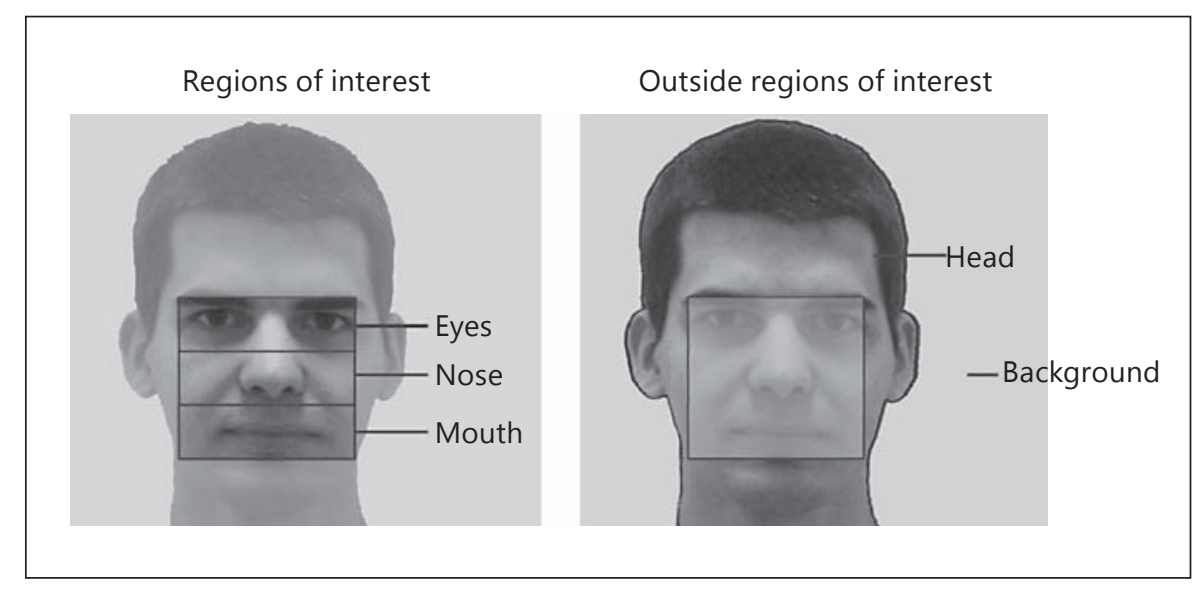

\section{Procedure}

Each of the 30 (color photograph) stimuli was presented for 4 $\mathrm{s}$ (interstimulus interval of $0.5 \mathrm{~s}$ with a black slide buffer) on a 21inch screen placed $80 \mathrm{~cm}$ in front of the subject. The choice of delivery during a 4-second period was validated in a pilot study in healthy adults and represents the window of time needed to obtain an exploration of the entire face. Moreover, being exposed to a neutral face for more than $5 \mathrm{~s}$ can provoke anxiety [19]. During the 4 -second period, the software recorded the eye position every $0.017 \mathrm{~s}$ (acquisition frequency: $60 \mathrm{~Hz}$ ). All stimuli were randomly presented to each participant. The participants had no instructions except to pay attention to the images and to remain silent during the experiment.

\section{Measurements}

Regions of interest were established in the regions of the eyes, nose, and mouth, and the exploration time and number of fixations on each region was calculated. Regions of interest were drawn around the eyes, nose, and mouth, which all have the same surface as described by Hernandez et al. [6] and Martineau et al. [8] (fig. 1). We also measured the exploration times of anything outside the regions of interest, i.e. the rest of the face (exploration time on the face excluding the eyes, nose, and mouth), the image background (exploration time on the details of the image around the face), and off the screen (exploration time outside the stimulation screen) (fig. 1).

\section{Statistical Analysis}

Analysis of the time spent on interest zones (eyes, nose, and mouth) according to the emotional expression of the face was done by repeated measurements using mixed ANOVA [4 (age group: $4-6$; 7-9; 10-12; 13-15; 18-35) × 2 (gender: male; female) $\times 3$ (zone of interest: eyes; nose; mouth)], corrected by the Greenhouse-Geisser test and followed by the Dunn-Bonferroni test to compare paired averages. Regression analysis was used to measure the effect of age on time spent on each area of interest.

\section{Results}

All subjects spent on average $93 \%$ of the time exploring the head (face and/or eyes and/or nose and/or mouth) and $7 \%$ of the time exploring the background.

\section{Effect of Age and Gender on Time Spent Looking}

On-Screen and Off-Screen

Analysis with mixed ANOVA revealed no gender effect $\left(F_{1,40}=0.128, p=0.72\right)$ on time spent looking on- or off-screen. Whatever the group and the emotions expressed by the face, a significant difference between time spent looking on- and off-screen was found $\mathrm{F}_{4,40}=$ $3,604.295$ ( $p<0.0001)$ : all groups spent more time looking on-screen $(3.72 \pm 0.36 \mathrm{~s}$, range: $2.35-4)$ than looking off-screen $(0.14 \pm 0.1 \mathrm{~s}$, range: $0.02-0.48, \mathrm{p}<0.0001)$. An effect of age $\left(\mathrm{F}_{4,40}=2.98, \mathrm{p}=0.03\right)$ and an interaction between age and the time spent looking on- and offscreen were $\mathrm{F}_{4,40}=5.663(\mathrm{p}<0.001)$ : children aged between 4 and 6 years spent less time looking on-screen $(3.42 \pm 0.43$ s, range: $2.35-3.90)$ than children aged between 10 and 12 years $(3.72 \pm 0.31 \mathrm{~s}$, range: $3.13-3.97$, $\mathrm{p}=0.04)$, children aged between 13 and 15 years $(3.90 \pm$ 0.16 s, range: $3.57-3.99, \mathrm{p}=0.001)$, and adults $(3.96 \pm$ 0.10 s, range: $3.58-4, p<0.0001)$, and children aged between 7 and 9 years spent less time looking on-screen $(3.63 \pm 0.37 \mathrm{~s}$, range: $2.54-4)$ than adults $(3.96 \pm 0.10 \mathrm{~s}$, range: $3.58-4, p=0.001)$. Regression analysis showed that all participants spent more time on-screen with age (4-6 years: $3.42 \pm 0.43$ s, range: $2.35-3.90$; $7-9$ years: $3.63 \pm$ 0.37 s, range: $2.54-4 ; 10-12$ years: $3.72 \pm 0.31 \mathrm{~s}$, range: $3.13-3.97$; $13-15$ years: $3.90 \pm 0.16 \mathrm{~s}$, range: $3.57-3.99$; adults: $3.96 \pm 0.10 \mathrm{~s}$, range: $3.58-4 \mathrm{~s})(\mathrm{p}<0.0001)$ and less time off-screen with age ( $4-6$ years: $0.24 \pm 0.13 \mathrm{~s}$, range: 
Table 2. Results of ANOVA comparing the different areas of interest of different stimuli in different age groups

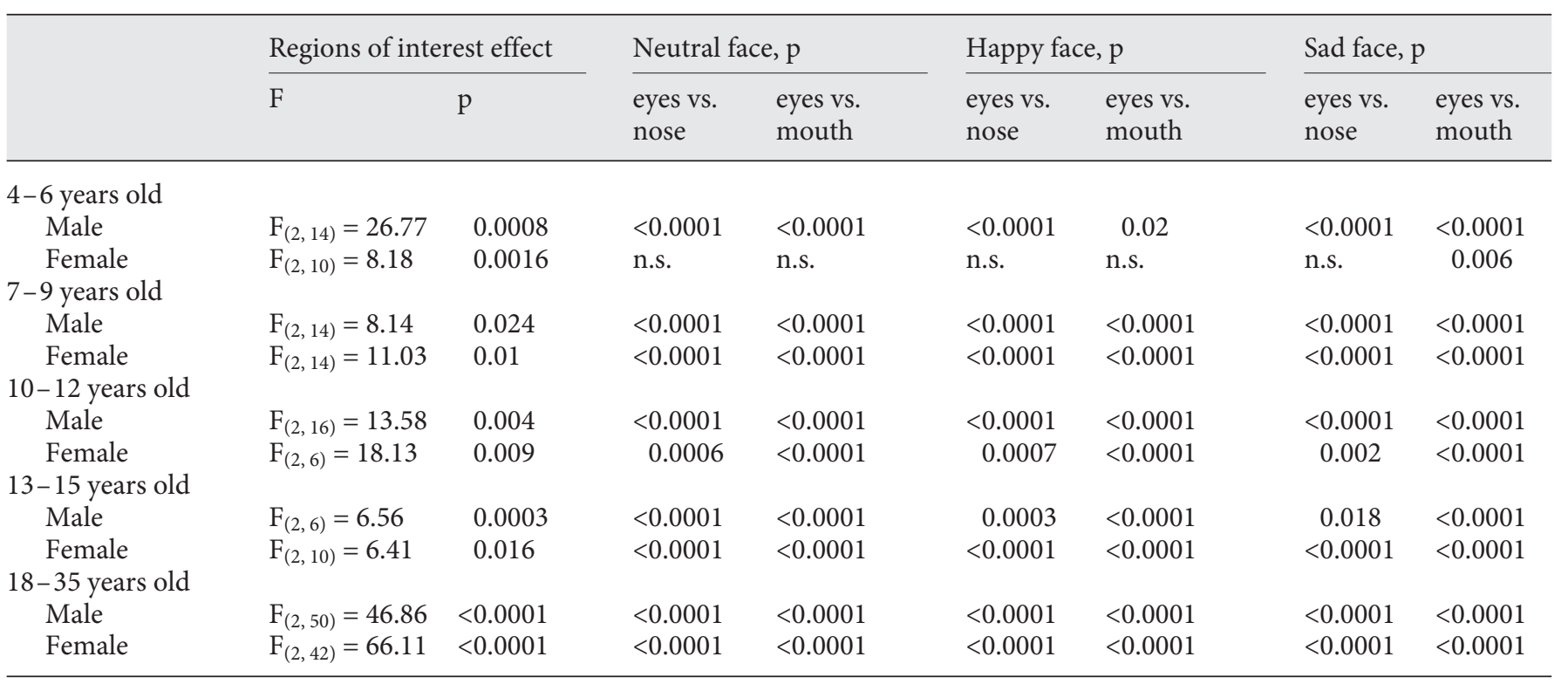

$0.07-0.48$; 7-9 years: $0.15 \pm 0.08$ s, range: $0.04-28 ; 10-12$ years: $0.11 \pm 0.06 \mathrm{~s}$, range: $0.05-0.29 ; 13-15$ years: $0.10 \pm$ $0.06 \mathrm{~s}$, range: $0.06-0.22$; adults: $0.07 \pm 0.07 \mathrm{~s}$, range: $0.02-$ $0.33)(\mathrm{p}<0.0001)$.

\section{Effect of Age and Gender on Time Spent Looking on the Regions of Interest}

Analysis with mixed ANOVA revealed a significant effect of the region of interest in participants in each gender group and age group $\left(\mathrm{F}_{2,182}=110.52, \mathrm{p}<0.0001\right)$ (table 2). Whatever the emotion expressed by the face, the time spent on the eye region was always significantly longer than the time spent on the nose and mouth regions in both females (eyes: $1.47 \pm 0.13 \mathrm{~s}$, range: $1.2-1.7$; nose: $0.46 \pm 0.08 \mathrm{~s}$, range: $0.25-0.67$; mouth: $0.21 \pm 0.09 \mathrm{~s}$, range: $0.09-0.4$ ) and males (eyes: $1.57 \pm 0.15 \mathrm{~s}$, range: $1.31-1.87$; nose: $0.54 \pm 0.1 \mathrm{~s}$, range: $0.36-0.73$; mouth: $0.25 \pm 0.07 \mathrm{~s}$, range: 0.14-0.4) (fig. 2). Mixed ANOVA revealed no effect of emotions expressed by the face in any of the groups (table 2). A gender effect was seen in children aged between 4 and 6 years $\left(\mathrm{F}_{1,40}=6.01, \mathrm{p}=0.019\right)$ and in children aged between 7 and 9 years $\left(\mathrm{F}_{1,46}=9.41, \mathrm{p}=0.004\right)$. Male children spent significantly more time $(\mathrm{p}=0.0002$ for boys aged between 4 and 6 years of age, and $p=0.008$ from 7 to 9 years of age) on the region of the eye (eyes: $1.32 \pm 0.36 \mathrm{~s}$, range: $0.57-2.08$ between 4 and 6 years of age and $1.68 \pm 0.41 \mathrm{~s}$, range: $0.68-2.56$ between 7 and 9 years of age) than female participants (eyes: $0.67 \pm 0.29 \mathrm{~s}$, range: $0.09-1.11$ between 4 and 6 years of age and $1.33 \pm$
0.38 s, range: $0.75-2.01$ between 7 and 9 years of age). No significant difference was observed in the time spent on the nose and mouth. This result was confirmed by the interaction between the age of development and gender $\left(\mathrm{F}_{4,293}=3.2, \mathrm{p}=0.013\right)$. No gender effects were observed in participants aged between 10 and 12 years $\left(\mathrm{F}_{1,37}=0.81\right.$, $\mathrm{p}=0.37)$, between 13 and 15 years $\left(\mathrm{F}_{1,28}=0.11, \mathrm{p}=0.74\right)$, or between 18 and 35 years $\left(\mathrm{F}_{1,142}=0.08, \mathrm{p}=0.77\right)$ (fig. 3 ). Regression analysis showed that female participants spent more time on the eye area with age $(\mathrm{p}<0.0001$, eyes: $0.67 \pm 0.29$ s, range: $0.09-1.11$ for girls aged $4-6$ years; $1.33 \pm 0.38 \mathrm{~s}$, range: $0.75-2.01$ for girls aged $7-9$ years; $1.82 \pm 0.46 \mathrm{~s}$, range: $1.17-2.88$ for girls aged $10-12$ years; $1.63 \pm 0.30 \mathrm{~s}$, range: $1.12-2.31$ for girls aged $13-15$ years, and $1.9 \pm 0.19 \mathrm{~s}$, range: $1.51-2.32$ for women; male participants, $\mathrm{p}<0.0001$, eyes: $1.32 \pm 0.36 \mathrm{~s}$, range: $0.57-2.08$ for boys aged $4-6$ years; $1.68 \pm 0.41 \mathrm{~s}$, range: $0.68-2.56$ for boys aged 7-9 years; $1.65 \pm 0.26 \mathrm{~s}$, range: $0.87-2.14$ for boys aged 10-12 years, eyes: $1.52 \pm 0.36$, for boys aged $13-15$ years, and $1.88 \pm 0.24 \mathrm{~s}$, range: $1.52-2.47$ for men). We found no significant differences in the time spent on the nose and mouth areas between the different age groups, neither in male nor female participants (fig. 3).

\section{Effect of Age and Gender on the Number of Fixations on the Regions of Interest}

Analysis with mixed ANOVA revealed no gender effect $\left(\mathrm{F}_{1,40}=0.866, \mathrm{p}=0.36\right)$. A significant effect of the region of interest on the number of fixations was found 


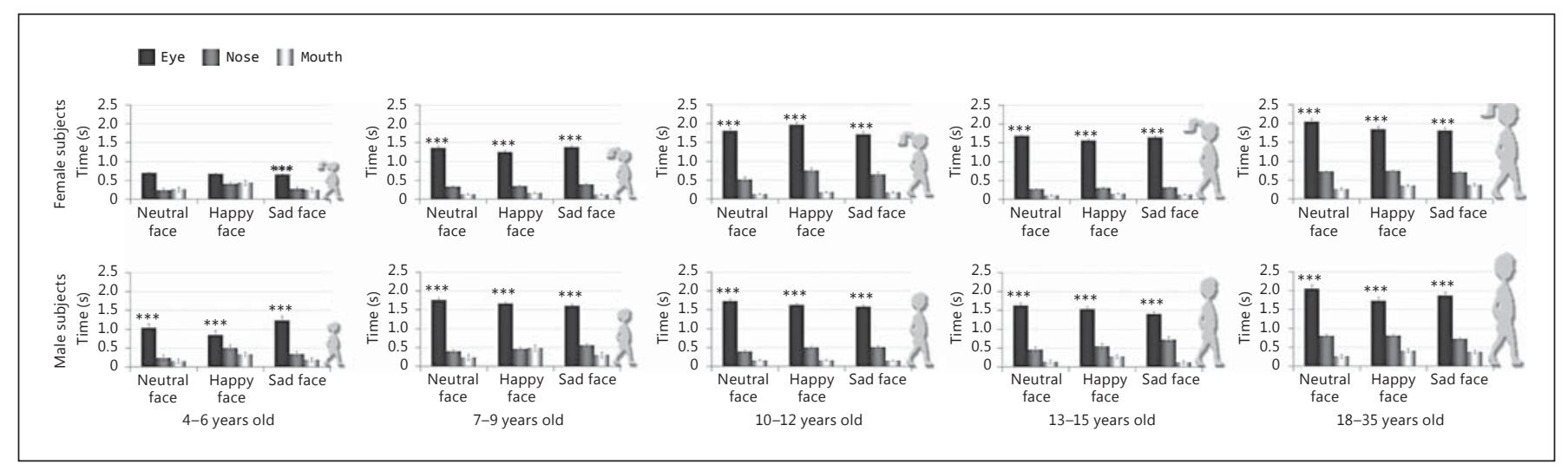

Fig. 2. Time spent on the different regions of interest. Mean and SD of time spent on the region of interest by male or female participants during development when they scanned neutral, happy, and sad faces. ${ }^{* * *} \mathrm{p}<0.0001$.

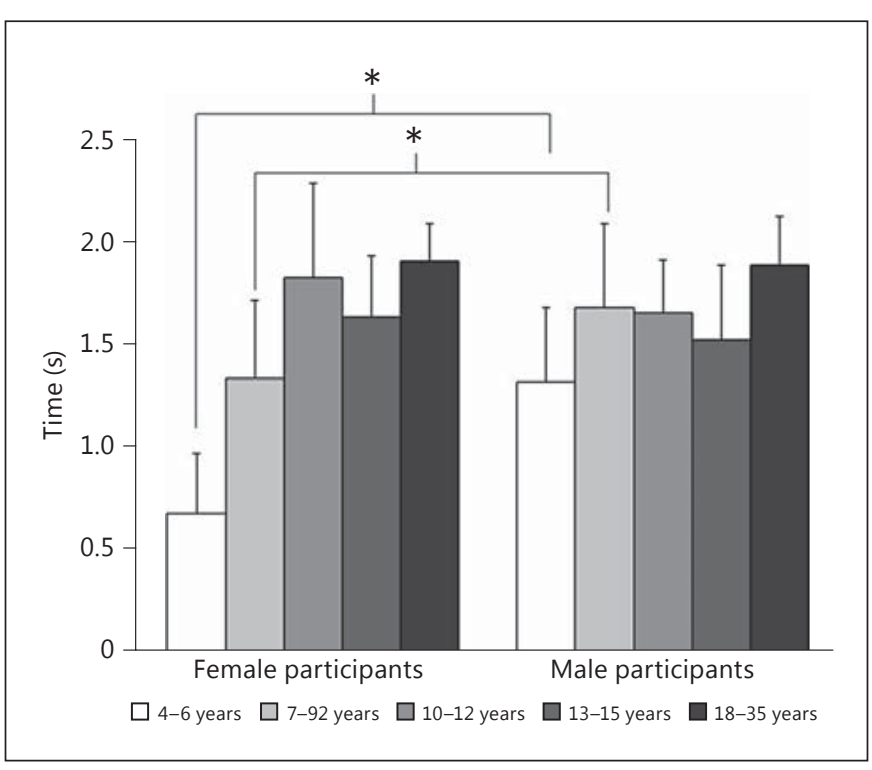

Fig. 3. Effect of development on the time spent on the eye region on the basis of gender. Mean and SD of time spent by male or female participants during development looking at the eye region of (neutral, happy, and sad) faces and the SD between male and female participants in the 4- to 6-year-old group and in the 7- to 9-year-old group. ${ }^{*} \mathrm{p}<0.05$.

$\left(\mathrm{F}_{2,182}=41.76, \mathrm{p}<0.0001\right)$ : whatever the emotion expressed by the face, the number of fixations on the eye region was always significantly higher than that on the nose and mouth regions (eyes: $8.15 \pm 3.38$; range: $1-19.07$; nose: $2.52 \pm 1.72$, range: $1-7.93$; mouth: $2.52 \pm 1.38$, range: $1-5.78)$. An effect of age on the number of fixations was found $\left(\mathrm{F}_{4,40}=4.48, \mathrm{p}=0.006\right)$ : children aged between 4 and 6 years realized globally fewer fixations than adults $(\mathrm{p}=0.003)$ : $(4-6$ years old: SD: 0.99 , range: $2.82-6.58$; adults: SD: 0.74 , range: 4.19-7.02). Regression analysis showed that whatever the emotion expressed by the face, the region of interest, and the gender of the participant, the number of fixations increased with age $(\mathrm{p}=0.006)$.

\section{Discussion}

In this study, the time spent exploring the stimulation was more important than the time spent off-screen, thereby indicating that the analysis was based on consistent time data that included younger children ( $4-6$ years old) who explored the images for $86 \%$ of the time. This exploration time increased with maturity (up to $99 \%$ of the time for adults), suggesting better attentional focus with age.

This study also demonstrated that whatever the age group, gender, or emotion expressed by the face, participants spent more time exploring and fixating the eye region. These findings confirmed previous reports $[6,7$, $20]$. Due to the wide range of information it conveyed regarding the internal state of the person, the eye region seemed to be the most important area when exploring the face. However, the presentation of a face expressing emotion could have diverted the attention of participants towards other areas involved in expressing emotions. This is exemplified in the exploration of a happy face that could produce an increase in the time spent on the mouth region. The findings indicated that the exploration of a face was not sensitive to morphological variations in the areas/parts of the face, and that the emotional information could be processed primarily at a cortical level, as at- 
tested by a number of functional studies (e.g. bilateral activation of the cingular gyrus by the expression of joy [21, 22 ] and lateralized activation on the left cingular gyrus by the expression of sadness [22]).

The study results also demonstrated an effect of gender on the time spent on the eye region. This gender effect was present in the children aged 4-10 years. At this age, male subjects spent more time exploring the eyes than female subjects. This result confirmed previous findings, thereby suggesting that there are gender differences in the development of sociocommunicative skills, such as language [10] and theory of mind [11]. Girls seemed to develop these skills earlier than boys, and most of these skills seemed ultimately better developed in women than in men (in particular skills such as level of empathy [23]). Beyond 10 years of age, exploration of the face was comparable in male and female subjects. The behavioral differences between the male and female subjects during the exploration of a face appeared to decrease with maturation. This finding could be related to the decrease in the cognitive activity's differences between men and women with maturation. Indeed, it had been shown that women have better verbal skills [24], whereas men have better visuospatial skills [25]. This difference between men and women is clearly present in youth and decreases with age [26].

Therefore, it is tempting to speculate that these differences in skills could be the origin of the behavioral differences observed in our study, which was related only to the time spent on the eye region, and not on the other regions of interest on the face (nose and mouth). This confirmed the importance of the eye region, and also underscored the fact that it was not simply a question of a difference in the exploration of the whole face, but an effect of gender on the perception of a socially relevant region. This could be plausible if we accept the assumption of the development of expertise in the perception of faces and their expressions, as shown by Carey [3]. Our finding suggested that maturation is accompanied by an increase in attention to a socially relevant region, i.e. the eyes, comparable to the development of maturation of expertise.

This study demonstrated an effect of maturation in both male and female participants exclusively regarding the increased attention to the eye region. It would seem that the time spent and the number of fixations on the eye region increased during the development irrespective of gender. More exactly, a shorter time was shown to have been spent on the eyes in the male participants of the youngest age group, and as early as 7-9 years of age it would seem that the time spent on the eye region was comparable to the time measured in adults, suggesting that male subjects reached mature exploratory behavior regarding faces at the age of 7-9 years. Comparable dynamics were observed for female participants, but the time spent on the eye region became comparable with that of adults only at the age of 10 years. This finding suggested that there is a shift between male and female subjects during development. Male subjects seemed to acquire mature behavior regarding the exploration of faces earlier than female subjects, apparently contradicting current beliefs of the social gender development [23, 27, 28 ]. Indeed, concerning the study of young children, female subjects tend to be more empathetic than male subjects [27] and they maintain eye contact very early on compared to male subjects [28].

There are 3 major limitations to our study: the static appearance of the stimuli, the low numbers in certain age groups, and the presentation of male faces only in the battery of stimuli. The static aspect of our stimuli could influence exploration of faces (in particular emotional faces) and must be taken into account in the interpretation of our results. Regarding age effect, our results should be interpreted with caution because of the small numbers of participants in a certain class of age (only 4 female participants between 10 and 12 years of age, and only 4 male participants between 13 and 15 years of age); however, our results on gender effect concerned the children aged 4-10 years, Finally, it is uncertain whether the gender differences observed in our study were linked to the different rates of development of the social skills in boys and girls or simply to the stimulus (of the male faces), which could be perceived differently by the female subjects. Because only male faces were presented in this study, it is possible that the male subjects were favored during the exploration of the stimuli, and it would be interesting to include women's faces in the database in a future study.

\section{Conclusion}

This study revealed an exploratory preference for the eye region compared to the nose and mouth when looking at a face. This preference was present regardless of age or gender, or of the emotion expressed by the face. But this preference seemed to establish gradually during maturation, with a shift between male and female subjects. Furthermore, the findings of this study underlined the need to study facial exploration separately based on age and gender (for children younger than 10 years) in future studies. 


\section{Acknowledgements}

We are grateful for the financial support of the Foundation for Medical Research, and the support of the INSERM Unit 930, the
University Hospital of Tours, the University of Tours François Rabelais, IFR 135, and the Planiol Foundation. We thank all the participants for their cooperation. We also thank Helen Dziri-Johnson for the final English revision of the text.

\section{References}

1 Turati C, Valenza E, Leo I, et al: Threemonth-olds' visual preference for faces and its underlying visual processing mechanisms. J Exp Child Psychol 2005;90:255-273.

-2 Burnham D: Visual recognition of mother by young infants: facilitation by speech. Perception 1993;22:1133-1133.

3 Carey S: Becoming a face expert. Philos Trans R Soc Lond B Biol Sci 1992;335:95-102, discussion 102-103.

4 Webb SJ, Long JD, Nelson CA: A longitudinal investigation of visual event-related potentials in the first year of life. Dev Sci 2005;8: 605-616.

5 Karatekin C: Eye tracking studies of normative and atypical development. Dev Rev 2007; 27:283-348

6 Hernandez N, Metzger A, Magné R, et al: Exploration of core features of a human face by healthy and autistic adults analyzed by visual scanning. Neuropsychologia 2009;47:10041012.

7 Klin A, Jones W, Schultz R, et al: Defining and quantifying the social phenotype in autism. Am J Psychiatry 2002;159:895-908.

-8 Martineau J, Hernandez N, Hiebel L, et al: Can pupil size and pupil responses during visual scanning contribute to the diagnosis of autism spectrum disorder in children? J Psychiatr Res 2011;45:1077-1082.

-9 Cui Y, Hondzinski JM: Gaze tracking accuracy in humans: two eyes are better than one. Neurosci Lett 2006;396:257-262.

10 Blakemore JEO, Berenbaum SA, Liben LS: Gender Development. Psychology Press, 2008, pp 80-82.
11 Walker S: Gender differences in the relationship between young children's peer-related social competence and individual differences in theory of mind. J Genet Psychol 2005;166: 297-312.

12 Barbu S, Cabanes G, Le Maner-Idrissi G: Boys and girls on the playground: sex differences in social development are not stable across early childhood. PLoS One 2011;6:e16407.

13 Cellerino A, Borghetti D, Sartucci F: Sex differences in face gender recognition in humans. Brain Res Bull 2004;63:443-449.

14 Thayer J, Johnsen BH: Sex differences in judgement of facial affect: a multivariate analysis of recognition errors. Scand J Psychol 2000;41:243-246.

15 Güntekin B, Basar E: Gender differences influence brain's beta oscillatory responses in recognition of facial expressions. Neurosci Lett 2007;424:94-99.

16 Oldfield RC: The assessment and analysis of handedness: the Edinburgh inventory. Neuropsychologia 1971;9:97-113.

17 Declaration of Helsinki. WMA Declaration of Helsinki - Ethical Principles for Medical Research Involving Human Subjects. FerneyVoltaire, World Medical Association, 2008.

18 Brooks CI, Church MA, Fraser L: Effects of duration of eye contact on judgments of personality characteristics. J Soc Psychol 1986; 126:71-78

19 Dalton KM, Nacewicz BM, Alexander AL, et al: Gaze-fixation, brain activation, and amygdala volume in unaffected siblings of individuals with autism. Biol Psychiatry 2007;61: 512-520.
20 Beltrán D, Calvo MG: Brain signatures of perceiving a smile: time course and source localization. Hum Brain Mapp 2015;36:42874303

21 Killgore WD, Yurgelun-Todd DA: Activation of the amygdala and anterior cingulate during nonconscious processing of sad versus happy faces. Neuroimage 2004;21:1215-1223.

22 Hasan S, Al-Sharqawi N, Dashti F, et al: Level of empathy among medical students in $\mathrm{Ku}$ wait University, Kuwait. Med Princ Pract 2013:22:385-389.

-23 Biele C, Grabowska A: Sex differences in perception of emotion intensity in dynamic and static facial expressions. Exp Brain Res 2006; 171:1-6.

24 Adelson J: What we don't know about sex differences. Const Comment 1986;3:295.

25 Halpern DF: Sex Differences in Cognitive Abilities. Psychology Press, 2000, p 198

26 Hoffman ML: Sex differences in empathy and related behaviors. Psychol Bull 1977;84:712722

27 Haviland JJ, Malatesta CZ: The development of sex differences in nonverbal signals: fallacies, facts, and fantasies; in Mayo C, Henley $\mathrm{N}$ (eds): Gender and Nonverbal Behavior. New York, Springer, 1981, pp 183-208.

28 Davidson H, Cave KR, Sellner D: Differences in visual attention and task interference between males and females reflect differences in brain laterality. Neuropsychologia 2000;38: 508-519. 